\title{
Endocardial/epicardial versus endocardial encircling cryoablation for ventricular tachycardia: A randomized trial
}

\author{
Carine F. van Huls van Taxis, MD, ${ }^{a}$ Katja Zeppenfeld, MD, PhD, ${ }^{a}$ Robert J. Klautz, MD, PhD, ${ }^{b}$ and \\ Adrianus P. Wijnmaalen, MD, PhD, ${ }^{a}$ Leiden, The Netherlands
}

\footnotetext{
From the Departments of ${ }^{\mathrm{a} C a r d i o l o g y}$ and ${ }^{\mathrm{b}}$ Cardiothoracic Surgery, Leiden University Medical Center, Leiden, The Netherlands.

Dr van Huls van Taxis receives funding from the Netherlands Heart Society (grant No. 2008B074).

Disclosures: Authors have nothing to disclose with regard to commercial support.

Received for publication March 16, 2018; revisions received Sept 14, 2018; accepted for publication Jan 3, 2019; available ahead of print Feb 26, 2019.

Address for reprints: Katja Zeppenfeld, MD, PhD, Cardiology Department, Leiden University Medical Center, Postbus 9600, 2300RC Leiden, The Netherlands (E-mail: K.Zeppenfeld@lumc.nl).

J Thorac Cardiovasc Surg 2019;157:e371-7

$0022-5223 / \$ 36.00$

Copyright (C) 2019 by The American Association for Thoracic Surgery

https://doi.org/10.1016/j.jtcvs.2019.01.012
}

Video clip is available online.

Patients remain at risk for ventricular tachycardia (VT) after surgical ventricular reconstruction. ${ }^{1-3}$ During surgery, concomitant preventive antiarrhythmic treatment may be of important incremental value. Standard nonmappingguided endocardial encircling cryoablation (ENDO) is unlikely to result in a complete transmural lesion in ventricular myocardium of normal wall thickness. ${ }^{4}$ We hypothesized that doubling the lesion depth by combined endocardialepicardial encircling cryoablation (ENDO-EPI) would lead to a transmural lesion electrically disconnecting the VT substrate from the remaining myocardium thereby preventing future VT. We aimed to determine the electrophysiological effects of ENDO-EPI cryoablation compared with ENDO cryoablation.

\section{METHODS}

The study was designed as a single-center, prospective, open-label parallel group comparison of conventional ENDO versus new combined ENDO-EPI (1:1 randomization) of the myocardial aneurysm scar borderzone to treat VT, approved by the institutional ethics committee (P07.257) and registered at the Netherlands Trial Register (No. NTR3340). The manuscript was prepared according to the Consolidated Standards of Reporting Trials checklist. Inclusion criteria were acceptance for surgical ventricular reconstruction and inducible aneurysm-related VT confirmed at electrophysiology study. Patients were included in our hospital from 2008 to 2011 by the treating physician and allocated according to the predefined randomization list.

During surgery, epicardial pacemaker lead pairs were placed at the left ventricle (LV), right ventricle, and the aneurysm (Figure 1). No subendocardial resection was performed. Cryoablation was performed with overlapping linear applications $(-145 \mathrm{C} ; 90$ seconds) at the endocardial aneurysm scar borderzone. ${ }^{5}$ In the ENDO-EPI group, additional

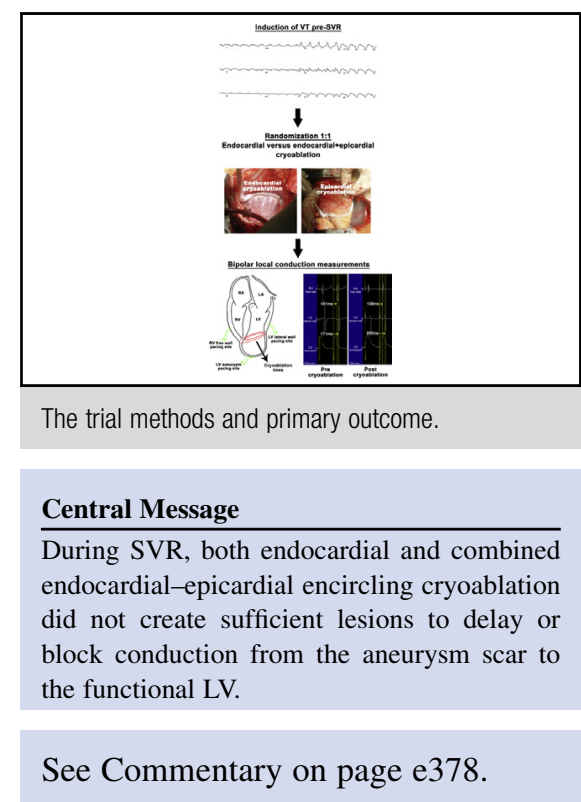

overlapping epicardial applications were applied opposite to the endocardial cryoablation line. Conduction properties were determined by pacing at all 3 pacemaker lead pairs before and after cryoablation.

The primary end point was the presence of $\geq 50 \mathrm{~ms}$ conduction delay or bidirectional block after cryoablation. The secondary end point was VT occurrence during follow-up. A detailed description is provided in Appendix E1. An overview of the trial is provided in Video 1.

\section{RESULTS}

Nine patients were enrolled to the ENDO group and 10 were enrolled to the ENDO-EPI group. One patient in the ENDO group withdrew from the study and was excluded from further analysis (see Tables E1 and E2 for patient and perioperative characteristics). Conduction over the cryoablation line during LV and right ventricle pacing was present in all without conduction delay. In 15 patients, pacing at the aneurysm resulted in capture and conduction without delay. In 3 patients (1 ENDO group patient and 2 ENDO-EPI group patients), pacing over the aneurysm lead pairs did not result in local capture; this was judged to be caused by manipulation of the leads during surgery. Two patients died of heart failure postoperatively (1 each group); 16 patients (7 ENDO group patients and 9 ENDO-EPI group patients) were discharged and prospectively followed. 


\section{Induction of VT pre-SVR}

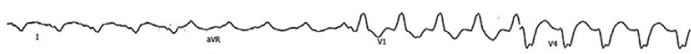

POn
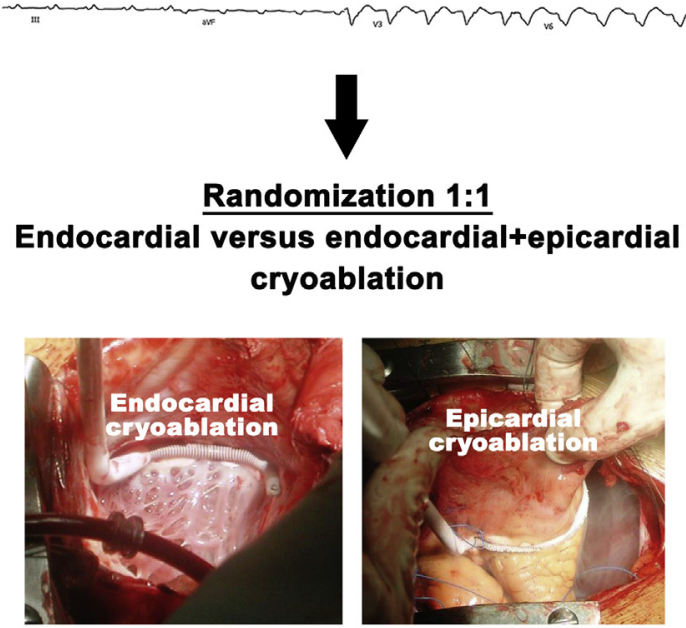

$\downarrow$

\section{Bipolar local conduction measurements}
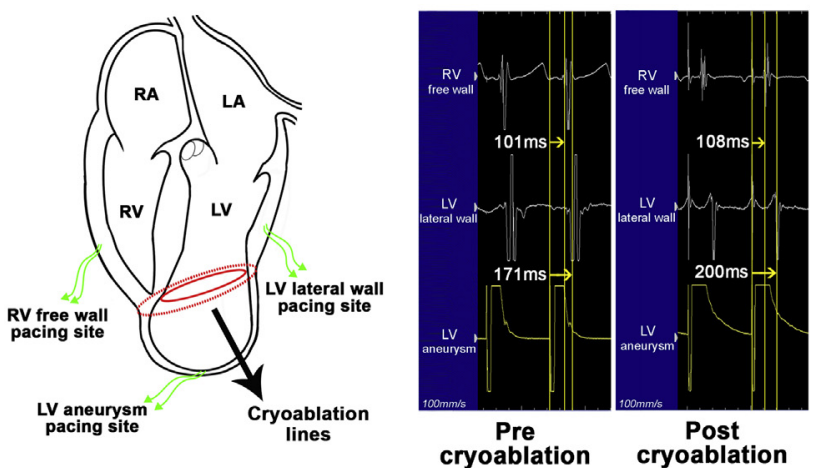

FIGURE 1. The trials' methods and primary outcome. Top, Induced ventricular tachycardia (VT) originating from the inferior-apical aneurysm borderzone. Middle, Flow chart of the 2 treatment arms. Left bottom, Schematic overview of pacemaker lead pairs placements in right ventricle (RV) and left ventricle (LV) and cryoablation lines. Right bottom, Example of conduction measurements with no significant conduction delay. $L A / R A$, Left and right atrium; SVR, surgical ventricular reconstruction.

The primary end point of a significant conduction delay or block was not established in any patient; therefore, after consultation with the institutional ethics committee it was decided to terminate the inclusion.

\section{Catheter ablation after SVR}

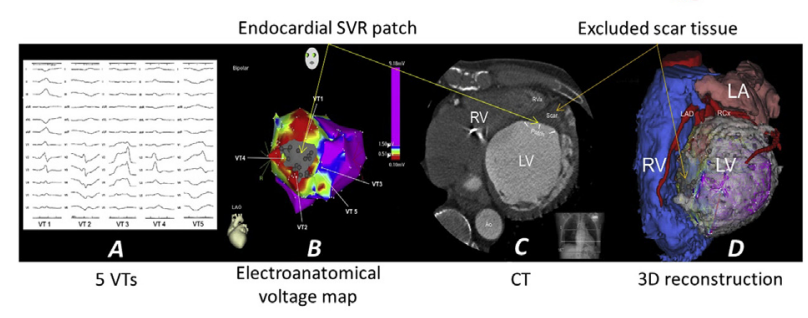

Excluded scar remains as substrate for VT

VIDEO 1. Presentation by Carine van Huls van Taxis explaining the relevance of the work. Video available at: https://www.jtcvs.org/article/S00225223(19)30063-7/fulltext.

VT occurred in 2 out of 7 patients in the ENDO group; both had multiple VTs in a brief period, 1 shortly after surgery (27 VTs) managed by catheter ablation and 1 year after (14 VTs) managed by sotalol. VT occurred in 5 out of 9 patients in the ENDO-EPI group 25 (range, 9-66) months after cryoablation (1-3 VT episodes/patients). Because of small numbers we could not evaluate differences between groups regarding the secondary end point.

\section{CONCLUSIONS}

During surgical ventricular reconstruction both ENDO and ENDO-EPI encircling cryoablation did not create sufficient lesions to significantly delay conduction from aneurysm scar to the functional LV. VT occurrence rate was high in both groups. Although the results are limited by the small number of patients studied, it appears encircling cryoablation did not prevent VT; therefore, other surgical techniques like endocardectomy should be considered.

\section{References}

1. Klein P, Bax JJ, Shaw LJ, Feringa HH, Versteegh MI, Dion RA, et al. Early and late outcome of left ventricular reconstruction surgery in ischemic heart disease. Eur J Cardiothorac Surg. 2008;34:1149-57.

2. Di Donato M, Sabatier M, Dor V, Buckberg G. Ventricular arrhythmias after LV remodelling: surgical ventricular restoration or ICD? Heart Fail Rev. 2004;9: 299-306.

3. van Huls van Taxis CF, Wijnmaalen AP, Klein P, Dekkers OM, Braun J, Verwey HF, et al. Programmed electrical stimulation-guided encircling cryoablation concomitant to surgical ventricular reconstruction for primary prevention of ventricular arrhythmias. Eur J Cardiothorac Surg. 2018;54: 98-105.

4. Guiraudon GM, Thakur RK, Klein GJ, Yee R, Guiraudon CM, Sharma A. Encircling endocardial cryoablation for ventricular tachycardia after myocardial infarction: experience with 33 patients. Am Heart J. 1994;128:982-9.

5. Wijnmaalen AP, Roberts-Thomson KC, Steven D, Klautz RJ, Willems S, Schalij MJ, et al. Catheter ablation of ventricular tachycardia after left ventricular reconstructive surgery for ischemic cardiomyopathy. Heart Rhythm. 2012;9:10-7. 


\section{APPENDIX E1: SUPPLEMENTAL METHODS}

The study was designed as a single-center, prospective, open-label parallel group comparison of endocardial versus combined endocardialepicardial cryoablation of myocardial scar borderzone to treat ventricular tachycardia (VT) that was approved by the institutional ethics committee between 2008 and 2011. Inclusion criteria were acceptation for surgical ventricular reconstruction (SVR) according to Dor $^{\mathrm{E}}{ }^{\mathrm{I}}$ in the Leiden University Medical Center, ischemic heart failure (New York Heart Association functional class 2-4), and age older than 18 years. Exclusion criteria were inability to comply with the protocol due to hemodynamic instability or to sign informed consent. The manuscript was prepared according to the Consolidated Standards for Reporting of Trials checklist.

\section{END POINTS}

The primary end point and assumed optimal acute electrophysiological outcome of nonmapping-guided encircling cryoablation was the electrical isolation of the scar area to exclude the arrhythmogenic part from the ventricles.

The normal left ventricular wall thickness at midventricular level is between $5.6 \pm 1.1 \mathrm{~mm}$ (minimum) and $9.5 \pm 1.9 \mathrm{~mm}$ (maximum) in men. ${ }^{\mathrm{E} 2}$ Because cryoablation has been shown to result in transmural lesions of $4 \mathrm{~mm}$ in the atrium and 6-mm lesion depth in ventricular myocardium, the creation of encircling transmural lesions on the scar border applying standard endocardial cryoablation is unlikely. ${ }^{\mathrm{E} 3 \mathrm{E} 4}$ In addition, our clinical observation showed that patients who underwent encircling cryoablation experienced VTs during follow-up to a similar rate as patients who did not undergo encircling cryoablation, indicating failure of the standard therapy to isolate the substrate in a large proportion of patients. It was therefore assumed that endocardial encircling cryoablation would result in isolation in a minority of patients. A high percentage of transmural lesions after combined endocardialepicardial cryoablation (doubling the lesion depth) was believed to be reasonable.

The secondary end point was occurrence of any sustained ventricular arrhythmia registered by the implantable cardioverter defibrillator (ICD) or electrocardiogram during follow-up.

\section{CLINICAL EVALUATION}

Before surgery, patients underwent extensive clinical evaluation consisting of careful history taking, echocardiography, coronary angiography, and ICD interrogation. The results, including functional status, presence of a left ventricular anterior-apical aneurysm, concomitant valve disease, and remaining coronary artery disease, were carefully evaluated by a team of cardiologists, electrophysiologists, and cardiothoracic surgeons according to the institutional protocol.

\section{Assessment of Myocardial Wall Thickness}

To evaluate whether the wall thickness assumed based on literature applied to the current patient population, diastolic wall thickness was measured at the healthy myocardium side of the scar border in the cine-magnetic resonance imaging images. Imaging planes and walls assessed were 4-chamber view: septal and lateral and 2-chamber view: anterior and inferior, short axis: 6 segments.

\section{Preoperative Electrophysiological Evaluation}

Two catheters were inserted through the right femoral vein, 1 placed at the His position and the second at the right ventricular apex and subsequently in the right ventricular outflow tract to perform preoperative electrophysiological evaluation (PES). The PES protocol consisted of 3 drive cycle lengths $(600,500$, and $400 \mathrm{~ms})$ with 1 to 3 ventricular extra stimuli (down to $200 \mathrm{~ms}$ or refractory period) and incremental burst pacing. An aneurysm-related VT substrate was assumed if PES induced a monomorphic VT, lasting $>30$ seconds or requiring termination because of hemodynamic compromise and the VT exit site was located at the aneurysm scar borderzone. The presumed exit site was determined based on the VT 12-lead electrocardiogram morphology. ${ }^{\mathrm{E} 5}$ All 12-lead VT electrocardiograms were analyzed by 2 independent observers. In case of discrepancy, agreement was reached by consensus. Patients with aneurysm-related VT were candidates for participation in the trial and asked to participate. After signing the informed consent paperwork, patients were randomly assigned in a 1:1 fashion to either the conventional surgical VT therapy of endocardial encircling cryoablation (ENDO group) to serve as a control group, or the newly proposed therapy of combined endocardial-epicardial encircling cryoablation (ENDO-EPI group).

\section{Surgical Technique}

Patients underwent SVR according to the previously described technique. ${ }^{\text {E1 }}$ Surgery was performed using cardiopulmonary bypass, aortic crossclamping, and intermittent warm blood cardioplegia. Epicardial temporary pacemaker lead-pairs were placed at the left ventricle and right ventricle, and the aneurysm. Then, the left ventricle was opened through the infarcted area. At the transitional zone between viable and scarred myocardium, endocardial encircling cryoablation was performed with a 4-mm diameter malleable cryoprobe (Cardioblate CryoFlex, Medtronic, Minneapolis, Minn) using argon gas. As mentioned above, the myocardium thickness targeted by encircling cryoablation is likely between 6 and $11 \mathrm{~mm}$. The available studies have mainly been performed in, thinner $\pm 4 \mathrm{~mm}$, atrial tissue where 60 seconds of cryoapplication $\left(-150^{\circ} \mathrm{C}\right)$ resulted in transmural lesions. Therefore we extended the freezing time by $50 \%$ to 90 seconds per application to achieve an assumed lesion depth of at least $6 \mathrm{~mm}$ in endocardial and $12 \mathrm{~mm}$ in combined endocardial and epicardial cryoablation. Overlapping linear 
applications, down to $-145^{\circ} \mathrm{C}$ for 90 seconds, were made to the aneurysm scar borderzone. In the ENDO-EPI group patients, additional overlapping epicardial cryoablation lesions were applied opposite to the endocardial cryoablation line.

After cryoablation, a Fontan stich was placed at the transitional zone. The residual left ventricle cavity was shaped and sized using a mannequin balloon (TRISVR; Chase Medical, Richardson, Tex) at $55 \mathrm{~mL} / \mathrm{m}^{2}$ body surface area and the remaining defect was closed through an endoventricular polyethylene terephthalate patch plasty. Excluded fibrous scar tissue was sutured over the patch to improve hemostasis. Additional concomitant procedures, such as valve surgery and/or coronary artery bypass grafting, were performed when indicated. After weaning the patient from extracorporeal circulation, transesophageal echocardiography was repeated to assess left ventricle shape and function, patch integrity, and valvular competency. Conduction properties between left ventricle, right ventricle, and aneurysm were measured by sequentially pacing at all 3 pacemaker lead-pairs consecutively before and after cryoablation.

No endocardial resection was performed during SVR in our cohort before this study. Prior investigation by Guiraudon and colleagues ${ }^{\mathrm{E} 6}$ demonstrated promising results by performing only encircling cryoablation concomitant to SVR. Therefore we adapted this strategy. Additionally, not resecting the myocardial scar has been for enhancement of surgical stability of the SVR and improved hemostasis during the procedure.

\section{ICD Settings}

In patients without an ICD before surgery, 1 was implanted before discharge based on the preoperative left ventricular ejection fraction $\leq 30 \%$ to $35 \%$ according to current European Society of Cardiology guidelines. Devices were programmed according to our standard institutional protocol for primary prevention; VT monitor zone (VT cycle length [VTCL] 321-400 ms, no therapy), VT zone (VTCL 261-320 ms, antitachycardia pacing) and if the VT continued ICD shocks), VF zone (VTCL $\leq 260 \mathrm{~ms}$, ICD shocks). Settings were adapted when clinically indicated.

\section{Follow-up}

Patients were prospectively followed in an outpatient heart failure program and maintained on optimal medical treatment for heart failure. ICDs were interrogated every 6 months. Printouts were reviewed for the occurrence of sustained VT, VTCL, and therapy mode. VT were classified as sustained when lasting $>30$ seconds in the ICD monitor zone or when initiated appropriate ICD therapy. Therapy was considered appropriate when occurring in response to any VT. Echocardiography was performed before discharge and afterward annually. The follow-up started at time of discharge and ended at last outpatient clinic visit up to June 2016 or death.

\section{Statistical Analysis}

We used a 2-independent-proportions power analysis to determine group size. It was assumed that $80 \%$ of patients in the study group and $30 \%$ in the control group would reach the primary outcome. Group sample sizes of 18 in the study group and 18 in the control group achieved a $91 \%$ power to detect a $50 \%$ difference between groups in regarding the primary end point. The test statistic used was the 2 -sided $Z$ test with pooled variance. The significance level of the test was targeted at $P=.05$.

Continuous variables are expressed as mean \pm standard deviation or median (interquartile range) and categorical variables as percentages, where appropriate. To compare groups for continuous variables student $t$ test (normally distributed data) and Mann-Whitney U test (nonnormally distributed data) were used. For categorical data, the Fisher exact test was used. General statistical analyses were performed using SPSS software version 22 (IBM-SPSS Inc, Armonk, NY). For the power calculation, PASS software was used (version 15, NCSS Statistical Software, Kaysville, Utah). The randomization list was constructed by $\mathrm{R}$ software (version 3.0.1, $\mathrm{R}$ Foundation for Statistical Computing, Vienna, Austria).

\section{RESULTS}

Table E1 describes patient characteristics and Table E2 describes perioperative characteristics.

\section{Assessment of Myocardial Wall Thickness}

Wall thickness was determined in a subset of 6 of 18 patients ( 2 in the ENDO group, 4 in the ENDO-EPI group) in whom preoperative magnetic resonance imaging data were available. In these patients, mean wall thickness of the borderzone was $7.9 \pm 1.6 \mathrm{~mm}$. Maximal wall thickness was found in the septal segments as was expected (mean, $8.7 \pm 1.7 \mathrm{~mm}$, maximal wall thickness measured $11.3 \mathrm{~mm}$ ).

\section{Programmed Electrical Stimulation After SVR}

Thirteen of 16 patients who were discharged underwent PES after SVR, using the same induction protocol as preoperatively. Two patients ( 1 both groups) died during the postoperative period before PES could be performed (due to heart failure). In 1 patient no PES was performed because of the preference of the patient (ENDO-EPI group) and the other 2 patients ( 1 both groups) experienced VT during the postoperative period so PES was believed to be unnecessary. Both these patients also experienced VTs during follow-up. Only 1 of 13 patients (ENDO group) who underwent programmed electrical stimulation after SVR was inducible for VT, which was aneurysm-related. During follow-up, this patient did not experience any VT. One other 
patient (from the ENDO group) was noninducible 1 week after surgery but experienced incessant VT 4 weeks later. This patient underwent successful radiofrequency catheter ablation where the clinical VT was terminated at the inferior-apical border of the patch.

\section{DISCUSSION}

\section{Limitations}

The study was designed as a prospective open label parallel group comparison. The nonblinded nature of the study may be associated with an inherent bias. Reliable blinding of those involved in performing the conduction measurements was considered to be unfeasible because these included the surgeon and those involved during the surgery. The treatment and measurements were done according to a standard protocol. Utmost care was taken to correctly assess the conduction before and after surgery.

The study was terminated after inclusion of 19 patients because the primary end point was not met in any of the participants. Because no histopathologic evaluation of the myocardial cryolesions was performed, it is not possible to determine the exact reason for failure of combined endocardial-epicardial cryoablation. Although VTs occurred in both groups, the resulting size of the study population is too small to make a relevant comparison between groups regarding the secondary end point (VT occurrence).

\section{E-References}

E1. Dor V, Saab M, Coste P, Kornaszewska M, Montiglio F. Left ventricular aneurysm: a new surgical approach. Thorac Cardiovasc Surg. 1989;37:11-9.

E2. Kawel N, Turkbey EB, Carr JJ, Eng J, Gomes AS, Hundley WG, et al. Norma left ventricular myocardial thickness for middle-aged and older subjects with steady-state free precession cardiac magnetic resonance: the multi-ethnic study of atherosclerosis. Circ Cardiovasc Imaging. 2012;5:500-8.

E3. Doll N, Kornherr P, Aupperle H, Fabricius AM, Kiaii B, Ullmann C, et al. Epicardial treatment of atrial fibrillation using cryoablation in an acute off-pump sheep model. Thorac Cardiovasc Surg. 2003;51:267-73.

E4. d'Avila A, Aryana A, Thiagalingam A, Holmvang G, Schmidt E, Gutierrez P, et al. Focal and linear endocardial and epicardial catheter-based cryoablation of normal and infarcted ventricular tissue. Pacing Clin Electrophysiol. 2008; 31:1322-31

E5. Miller JM, Marchlinski FE, Buxton AE, Josephson ME. Relationship between the 12-lead electrocardiogram during ventricular tachycardia and endocardial site of origin in patients with coronary artery disease. Circulation. 1988;77: 759-66.

E6. Guiraudon GM, Thakur RK, Klein GJ, Yee R, Guiraudon CM, Sharma A. Encircling endocardial cryoablation for ventricular tachycardia after myocardial infarction: experience with 33 patients. Am Heart J. 1994;128:982-9. 
TABLE E1. Baseline characteristics

\begin{tabular}{|c|c|c|c|c|}
\hline Characteristic & $\begin{array}{l}\text { Total population } \\
\quad(\mathbf{N}=\mathbf{1 8}) \\
\end{array}$ & $\begin{array}{l}\text { ENDO group } \\
\quad(\mathbf{n}=\mathbf{8})\end{array}$ & $\begin{array}{l}\text { ENDO-EPI group } \\
\qquad(\mathbf{n}=\mathbf{1 0})\end{array}$ & $P$ value* \\
\hline Male & $15(83)$ & $6(75)$ & $9(90)$ & $.559 \dagger$ \\
\hline Age $(y)$ & $64 \pm 10$ & $62 \pm 9$ & $66 \pm 11$ & $.390 \ddagger$ \\
\hline LV ejection fraction $(\%)$ & $27 \pm 6$ & $27 \pm 5$ & $27 \pm 6$ & $.979 \ddagger$ \\
\hline LV end diastolic volume index & $112 \pm 44$ & $129 \pm 58$ & $102 \pm 34$ & $.230+$ \\
\hline $\mathrm{LV}$ end systolic volume index $\left(\mathrm{mL} / \mathrm{m}^{2}\right)$ & $82 \pm 36$ & $97 \pm 49$ & $74 \pm 25$ & $.260 \ddagger$ \\
\hline Diabetes mellitus & $5(27)$ & 0 & $5(50)$ & $.036 \dagger$ \\
\hline Atrial fibrillation & $4(22)$ & $1(13)$ & $3(33)$ & $.588 \dagger$ \\
\hline \multicolumn{5}{|l|}{ Medication } \\
\hline Beta-blocker & $18(100)$ & $8(100)$ & $10(100)$ & $1.0^{\dagger}$ \\
\hline ACE inhibitor/angiotensin II receptor blockers & $18(100)$ & $8(100)$ & $10(100)$ & $1.0 \dagger$ \\
\hline Mineralocorticoid receptor antagonists & $11(61)$ & $4(50)$ & $7(70)$ & $.630 \dagger$ \\
\hline Antiarrhythmic drugs & $4(22)$ & 0 & $4(40)$ & $.092 \dagger$ \\
\hline New York Heart Association functional class & & & & $1.000^{\dagger}$ \\
\hline 2 & $8(44)$ & $4(50)$ & $4(40)$ & \\
\hline 3 & $10(66)$ & $4(50)$ & $6(60)$ & \\
\hline Creatinine clearance $\left(\mathrm{mL} / \mathrm{min} \times 1.73 \mathrm{~m}^{2}\right)$ & $72 \pm 27$ & $70 \pm 20$ & $73 \pm 33$ & $.812 \ddagger$ \\
\hline EuroSCORE & $10(5-24)$ & $6(4-20)$ & $19(8-25)$ & $.203 \S$ \\
\hline Coronary artery disease & $1.9 \pm 0.7$ & $1.9 \pm 0.8$ & $1.9 \pm 0.6$ & $.941 \ddagger$ \\
\hline Left anterior descending artery & $18(100)$ & $8(100)$ & $10(100)$ & \\
\hline Right coronary artery & $9(50)$ & $6(75)$ & $3(30)$ & \\
\hline Ramus circumflexus & $7(39)$ & $4(50)$ & $3(30)$ & \\
\hline $\begin{array}{l}\text { Time from myocardial infarction to surgical ventricular } \\
\text { reconstruction (mo) }\end{array}$ & $53(10-213)$ & $51(12-170)$ & $53(5-294)$ & $.762 \S$ \\
\hline Characteristics induced VT & 22 & 10 & 12 & \\
\hline VTs/patient & $1.2 \pm 0.5$ & $1.3 \pm 0.5$ & $1.2 \pm 0.6$ & $.854 \ddagger$ \\
\hline Cycle length & $267 \pm 56$ & $267 \pm 63$ & $267 \pm 53$ & $.961 \ddagger$ \\
\hline Left axis & $14(64)$ & $7(70)$ & $7(58)$ & $.380^{\dagger}$ \\
\hline Left bundle branch block & $10(46)$ & $5(50)$ & $5(42)$ & $.666+$ \\
\hline
\end{tabular}

Values are presented as $\mathrm{n}(\%)$, mean \pm standard deviation, or median (interquartile range). ENDO, Standard endocardial encircling cryoablation; EPI, combined endocardialepicardial encircling cryoablation; $L V$, left ventricle; $A C E$, angiotensin-converting enzyme; EuroSCORE, European system for cardiac operative risk evaluation; VT, ventricular tachycardia. ${ }^{*} P$ value calculated between groups. †Fisher exact test. $\ddagger$ Student $t$ test. $§ M a n n$ Whitney $U$ test. 
TABLE E2. Perioperative characteristics

\begin{tabular}{|c|c|c|c|c|}
\hline Characteristic & $\begin{array}{c}\text { Total population } \\
\quad(\mathbf{N}=\mathbf{1 8}) \\
\end{array}$ & $\begin{array}{l}\text { ENDO group } \\
\quad(n=8)\end{array}$ & $\begin{array}{l}\text { ENDO-EPI group } \\
\quad(n=10)\end{array}$ & $P$ value* \\
\hline Coronary artery bypass grafting & $12(67)$ & $5(63)$ & $7(70)$ & $.737 \dagger$ \\
\hline Mitral valve plasty & $9(50)$ & $3(38)$ & $6(60)$ & $.343 \ddagger$ \\
\hline Tricuspid valve plasty & $4(22)$ & $1(13)$ & $3(30)$ & $.375 \ddagger$ \\
\hline Aortic valve replacement & $1(11)$ & $1(13)$ & 0 & $.250 \ddagger$ \\
\hline Cardiopulmonary bypass time (min) & $212 \pm 67$ & $200 \pm 75$ & $222 \pm 62$ & $.509 \dagger$ \\
\hline Aortic crossclamping time (min) & $135 \pm 47$ & $126 \pm 62$ & $142 \pm 39$ & $.516 \dagger$ \\
\hline Postoperative stay (d) & $11(8-15)$ & $11(8-14)$ & $11(9-18)$ & $.573 \S$ \\
\hline Postoperative mortality & $2(11)$ & $1(13)$ & $1(10)$ & $1.0 \ddagger$ \\
\hline \multicolumn{5}{|l|}{ Postoperative echocardiography } \\
\hline LV ejection fraction $(\%)$ & $32 \pm 12$ & $35 \pm 6$ & $30 \pm 15$ & $.429 \dagger$ \\
\hline $\mathrm{LV}$ end diastolic volume index $\left(\mathrm{mL} / \mathrm{m}^{2}\right)$ & $77 \pm 17$ & $73 \pm 16$ & $79 \pm 19$ & $.514 \dagger$ \\
\hline $\mathrm{LV}$ end diastolic volume index $\left(\mathrm{mL} / \mathrm{m}^{2}\right)$ & $48 \pm 17$ & $46 \pm 12$ & $50 \pm 20$ & $.626 \dagger$ \\
\hline
\end{tabular}

Values are presented as $\mathrm{n}(\%)$, mean \pm standard deviation, or median (interquartile range). ENDO, Standard endocardial encircling cryoablation; $E P I$, combined endocardialepicardial encircling cryoablation; $L V$, left ventricle. ${ }^{*} P$ value calculated between groups. $\dagger$ Student $t$ test. †Fisher exact-test. §Mann-Whitney U test. 